CERN-TH.6436/92

IEM-FT-53/92

\title{
SUPERSYMMETRY BREAKING AND DETERMINATION OF THE UNIFICATION GAUGE COUPLING CONSTANT IN STRING THEORIES
}

\author{
B. de CARLOS*, J.A. CASAS ${ }^{* * * *}$ and C. MUÑOZ** \\ * Instituto de Estructura de la Materia (CSIC), \\ Serrano 123, 28006-Madrid, Spain \\ ** CERN, CH-1211 Geneva 23, Switzerland
}

\begin{abstract}
We study in a systematic and modular invariant way gaugino condensation in the hidden sector as a potential source of hierarchical supersymmetry breaking and a non-trivial potential for the dilaton $S$ whose real part corresponds to the tree level gauge coupling constant ( $\operatorname{Re} S \sim g_{g u t}^{-2}$ ). For the case of pure Yang-Mills condensation, we show that no realistic results (in particular no reasonable values for $\operatorname{Re} S$ ) can emerge, even if the hidden gauge group is not simple. However, in the presence of hidden matter (i.e. the most frequent case) there arises a very interesting class of scenarios with two or more hidden condensing groups for which the dilaton dynamically acquires a reasonable value (Re $S \sim 2)$ and supersymmetry is broken at the correct scale $\left(m_{3 / 2} \sim 10^{3} \mathrm{GeV}\right)$ with no need of fine-tuning. Actually, good values for $\operatorname{Re} S$ and $m_{3 / 2}$ are correlated. We make an exhaustive classification of the working possibilities. Remarkably, the results are basically independent from the value of $\delta^{G S}$ (the contributions from the Green-Schwarz mechanism). The radius of the compactified space also acquires an expectation value, breaking duality spontaneously.
\end{abstract}

CERN-TH.6436/92

IEM-FT-53/92

March 1992 



\section{Introduction}

Recent LEP measurements corroborate [1] within a high level of accuracy the expectations of supersymmetric grand unification [2]. Namely, the gauge coupling constants of the standard model seem to be unified at $M_{\text {gut }} \sim 10^{16} \mathrm{GeV}$ with a value $\alpha_{\text {gut }}=g_{\text {gut }}^{2} / 4 \pi \sim$ $1 / 26$. This unification arises naturally in the context of superstring theories, where all the couplings are essentially the same at tree level, even in the absence of a grand unification group. Nevertheless there remains the fundamental question of why $g_{\text {gut }}$ should have any particular value. (Note that this problem cannot even be posed in the framework of an ordinary GUT, where $g_{g u t}$ is an input parameter.) If superstring theories are the fundamental theory from which the standard model is derived as a low energy limit, they should be able to give an answer to the previous question. In fact this is mandatory since in superstring theories we do not have the freedom to introduce the fundamental constants as input parameters. In particular the tree level gauge coupling constant corresponds to the real part of a complex scalar field, the dilaton $S\left(\operatorname{Re} S \equiv S_{R} \sim g_{\text {gut }}^{-2}\right.$ ), so experimental data demand $S_{R} \sim 2$. Therefore the initial question translates as how is the vacuum expectation value (VEV) of $S$ determined. Unfortunately $S$ has a flat scalar potential at all order in string perturbation theory [3], at least while supersymmetry remains unbroken. This also applies to the moduli fields $T_{i}$, which are singlet under gauge interactions and whose VEVs are usually associated with the size and shape of the compactified space. Actually one does not have even the choice of assuming that $\langle S\rangle,\left\langle T_{i}\right\rangle$ are given at present by their own cosmological evolution since the strong bounds on the variation of the gauge coupling constants with time and on scalar-tensor theories of gravity exclude this possibility [4]. Therefore, non-perturbative effects must play a fundamental role in the determination of $\langle S\rangle,\left\langle T_{i}\right\rangle$.

As has been suggested above this process should be strongly related to the supersymmetry breaking mechanism. This is by itself one of the most important technical problems which remains unsolved in superstring theories. More precisely, it is not yet understood how supersymmetry can break at a scale hierarchically smaller than the Planck scale $M_{P}$, which is essential for correct low energy physics. The most promising source of non-perturbative effects capable of generating this hierarchical supersymmetry breaking is gaugino condensation in the hidden sector [5]. The reason for this is that the scale of gaugino condensation corresponds to the scale at which the gauge coupling becomes large and this is governed by the running of the coupling constant. Since the running is only logarithmically dependent on the scale, the gaugino condensation scale is suppressed

relative to the initial one by an exponentially small factor $\sim e^{-\frac{1}{2 \beta g^{2}}}$ providing a promising 
source for the mass hierarchy ( $\beta$ is the one-loop coefficient of the beta function of the hidden sector gauge group $G$ ). However in string theories it is not obvious this mechanism will work for, as mentioned above, the value of the gauge coupling at the starting scale for evolution is itself a dynamical variable. It should be mentioned here that these are effects in the low energy field theory. At present there is no framework for calculating non-perturbative effects in the full string theory, so one has to assume that field theory non-perturbative effects dominate over the stringy ones. On the other hand this seems reasonable since we are examining the physics at low energy, where quantum field theory is a good approximation of string theory.

Much work has recently been devoted to the study of gaugino condensation in the framework of string theories [6-14]. It has been learned that the assumption of duality, i.e. target space modular $S L(2, Z)$ symmetry in the effective four dimensional supergravity action [15], modifies the usual form of the condensate [8-10] in essential agreement with previous one-loop calculations including string heavy modes [16]. This modification, which has been slightly corrected in ref.[14] by taking into account the contributions from the Green-Schwarz mechanism $[17,18]$, has proved to be extremely useful to fix the VEVs of some of the moduli $T_{i}[8,9]$. It has also been shown that no further modifications arise from higher string loops [19]. The influence of hidden matter, which is usually present, in the condensation process has also been studied [7,11-14,20]. However, in spite of all these achievements there are no known scenarios working satisfactorily up to now. In particular, reasonable values for the dilaton (and thus for the gauge coupling constant) have proved to be very difficult to obtain. It has been suggested that the existence of two or more condensates in the hidden sector $[21,6,7,22]$, as well as the presence of hidden matter $[7,12]$, may contribute to fixing the dilaton correctly and breaking supersymmetry at the right scale. However, no systematic analysis of these possibilities has been performed yet. This is precisely the main goal of this paper.

Our starting point is the effective field theory coming from a compactified string theory leaving one supersymmetry intact. The resulting $D=4 N=1$ supergravity theory [23] is characterized by the superpotential $W$ and the gauge kinetic function $f$, holomorphic in the chiral superfields, and the Kähler potential $K$, real-analytic in the chiral superfields. These functions are determined, in principle, in a given compactification scheme, although in practice they are known completely only for orbifold compactification schemes [24], which on the other hand have proved to possess very attractive features

${ }^{1} \beta=\frac{1}{16 \pi^{2}}\left(3 C(G)-\sum_{R} T(R)\right)$, where $C(G)$ is the Casimir operator in the adjoint representation of $G, R$ labels the chiral supermultiplets transforming like representations of $G$ and $T(R)$ is defined by $\operatorname{Tr}\left(T^{i} T^{j}\right)=T(R) \delta^{i j}$. 
from the phenomenological point of view [25]. More precisely, in the general case when the gauge group contains several factors $G=\prod_{a} G_{a}$, the exact gauge kinetic functions in string perturbation theory, up to small field-independent contributions, are [16-18]

$$
f_{W}^{a}=k^{a} S+\frac{1}{4 \pi^{2}} \sum_{i=1}^{3}\left(\frac{1}{2} b_{i}^{a}-k^{a} \delta_{i}^{G S}\right) \log \left(\eta\left(T_{i}\right)\right)^{2},
$$

with

$$
b_{i}^{\prime a}=C\left(G^{a}\right)-\sum_{Q} T\left(R_{Q}^{a}\right)\left(1+2 n_{Q}^{i}\right),
$$

where the meaning of the various quantities appearing in (1,.2) is the following: $k^{a}$ is the Kac-Moody level of the $G^{a}$ group ( $k^{a}=1$ is a very common possibility), $T_{i}$ ( $i=$ $1,2,3)$ are untwisted moduli, whose real parts give the radii of the three compact complex dimensions of the orbifold $\left(\operatorname{Re} T_{i}=R_{i}^{2}, i=1,2,3\right), \delta_{i}^{G S}$ are 1-loop contributions coming from the Green-Schwarz mechanism, which have been determined for the simplest $(2,2)$ $Z_{N}$ orbifolds [17], $\eta\left(T_{i}\right)$ is the Dedekind function, $Q$ labels the matter fields transforming as $R_{Q}^{a}$ representations under $G^{a}$ and $n_{Q}^{i}$ are the corresponding modular weights (see below). The 1-loop Kähler potential, up to terms involving matter fields, is [17]

$$
\begin{aligned}
K_{1-\text { loop }} & =-\log \left(S+\bar{S}+\frac{1}{4 \pi^{2}} \sum_{i=1}^{3} \delta_{i}^{G S} \log \left(T_{i}+\bar{T}_{i}\right)\right)-\sum_{i=1}^{3} \log \left(T_{i}+\bar{T}_{i}\right) \\
& =-\log Y-\sum_{i=1}^{3} \log \left(T_{i}+\bar{T}_{i}\right) .
\end{aligned}
$$

The function $Y=S+\bar{S}+\frac{1}{4 \pi^{2}} \sum_{i} \delta_{i}^{G S} \log \left(T_{i}+\bar{T}_{i}\right)$ can be considered [17] as the redefined gauge coupling constant at the unifying string scale: $Y=\frac{2}{g_{\text {string }}^{2}}$. Finally, the perturbative superpotential $W^{\text {pert }}$ has the form

$$
W^{p e r t}=h_{I J K} Q_{I}^{(u)} Q_{J}^{(u)} Q_{K}^{(u)}+h_{I J K}^{\prime}\left(T_{i}\right) Q_{I}^{(t)} Q_{J}^{(t)} Q_{K}^{(t)}+h_{I J K}^{\prime \prime} Q_{I}^{(u)} Q_{J}^{(t)} Q_{K}^{(t)}+\cdots,
$$

where $Q_{I}^{(u)}\left(Q_{I}^{(t)}\right)$ are untwisted (twisted) charged matter fields. The value of $h_{I J K}, h_{I J K}^{\prime \prime}$ for the allowed couplings is simply one, while $h_{I J K}^{\prime}\left(T_{i}\right)$ are complicated but known functions of $T_{i}[26,27]$. The dots in (4) stand for non-renormalizable terms and other terms involving twisted moduli which are not relevant for the discussion here. We will make use of the previous expressions throughout the paper.

As mentioned above the theory is invariant under $[S L(2, Z)]^{3}$ target space modular transformations, which are of the form

$$
T_{i} \rightarrow \frac{a_{i} T_{i}-i b_{i}}{i c_{i} T_{i}+d_{i}}, \quad a_{i} d_{i}-b_{i} c_{i}=1, \quad a_{i}, b_{i}, c_{i}, d_{i} \in Z
$$


This means in particular that the combination $\mathcal{G} \equiv K+\log |W|^{2}$ must be modular invariant which, using (3), implies that the superpotential has to transform with modular weight -3 [15], i.e.

$$
W \quad \rightarrow \quad W \prod_{i=1}^{3}\left(i c_{i} T_{i}+d_{i}\right)^{-1}
$$

and the dilaton $S$ has to transform as [17]

$$
S \quad \rightarrow \quad S+\frac{1}{4 \pi^{2}} \sum_{i=1}^{3} \delta_{i}^{G S} \log \left(i c_{i} T_{i}+d_{i}\right)
$$

Note that the function $Y$ defined in (3) is modular invariant. Similarly, charged matter fields $Q$ transform as

$$
Q \quad \rightarrow \quad Q \prod_{i=1}^{3}\left(i c_{i} T_{i}+d_{i}\right)^{n_{Q}^{i}}
$$

In particular, untwisted matter fields associated with the $j$-th complex plane have modular weights $n_{Q}^{i}=-\delta_{j}^{i}$. Modular weights corresponding to various types of twisted matter fields can be found in ref.[28]. The dependence of $h_{I J K}, h_{I J K}^{\prime}, h_{I J K}^{\prime \prime}$ on $T_{i}$ is such that $W^{\text {pert }}$ has modular weight -3 .

In section 2 the case of pure Yang-Mills gaugino condensation in the hidden sector is analysed in a systematic way for one, two or more condensing subsectors taking the $\delta^{G S}=0$ case as a guide example and extending afterwards the results to $\delta^{G S} \neq 0$. We show that no realistic results (in particular no reasonable values for $S_{R}$ ) can emerge in this context, even if the the hidden sector gauge group is not simple. In section 3 we consider the most frequent case, namely gaugino condensation in the presence of matter. We again take $\delta^{G S}=0$ and matter of the untwisted type as a useful guide example, eventually extending the results to the most general case, i.e. $\delta^{G S} \neq 0$ and matter in the twisted sector. In this way there arises a wide and very interesting class of scenarios with two hidden condensing groups $S U\left(N_{1}\right) \times S U\left(N_{2}\right)$ for which the dilaton dynamically acquires a reasonable value $\left(S_{R} \sim 2\right)$ and supersymmetry is broken at the correct scale $\left(m_{3 / 2} \sim 10^{3} \mathrm{GeV}\right)$. Actually, good values for $\operatorname{Re} S$ and $m_{3 / 2}$ turn out to be correlated. We have made an exhaustive analysis of the working possibilities (see tables), including also Kac-Moody levels $k \neq 1$ and other scenarios with more than two condensing groups. In section 4 we present our conclusions. 


\section{Gaugino condensation without matter}

\subsection{General aspects}

Examples of pure Yang-Mills (YM) hidden sectors in superstring constructions can be found in the literature. For instance, the $E_{8}^{\prime}$ gauge group for the standard embedding of $Z_{N}$ and $Z_{N} \times Z_{M}$ orbifolds is well known. Also $Z_{7}, Z_{4}$ models with $S U(3) \times S U(2) \times U(1)^{5}$ observable sector and pure $E_{6}, S U(3) \times S U(3)$ gauge hidden sectors respectively were constructed [29] and many more examples can be built up.

The process of gaugino condensation in the context of a pure Yang-Mills (YM) $N=1$ supergravity theory has been pretty well understood for a long time [5]. It can be conveniently described [9] by an effective superpotential $W^{n p}(U)$ of the chiral composite superfield $U\left(=\delta_{a b} W_{\alpha}^{a} \epsilon^{\alpha \beta} W_{\beta}^{b}\right)$ whose scalar component corresponds to the gaugino composite bilinear field $(\lambda \lambda)$. $W^{n p}$ reads

$$
W^{n p}=\frac{U}{4}\left(f_{W}+\frac{2}{3} \beta \log U\right) .
$$

In our case $f_{W}$ is given by eq.(11) with $b_{i}^{\prime}=16 \pi^{2} \beta / 3$. As it has been demonstrated in refs. $[7,11,12]$, it is equivalent to work with (9) than with the resulting superpotential after substituting $\partial W^{n p} / \partial U=0$, i.e.

$$
W^{n p}=d e^{-\frac{3}{2 \beta} f_{W}}=d \frac{e^{-\frac{3 k}{2 \beta} S}}{\prod_{i=1}^{3}\left[\eta\left(T_{i}\right)\right]^{\left(2-\frac{3 k}{4 \pi^{2} \beta} \delta_{i}^{G S}\right)}},
$$

where $d=-\beta / 6 e$. Notice that in the previous expression the form of $f_{W}$ is valid at all order in perturbation theory [19]. If the gauge group is not simple, $G=\prod_{a} G_{a}$, then $W^{n p}=\sum_{a} W_{a}^{n p}$. Using the Kähler potential of eq.(3), the scalar potential (valid for a generic $W(S, T))$ is given by $[14]$

$$
\begin{aligned}
V= & \frac{1}{Y \prod_{i=1}^{3}\left(T_{i}+\bar{T}_{i}\right)}\left\{\left|Y W_{S}-W\right|^{2}+\sum_{i=1}^{3} \frac{Y}{Y+\frac{1}{4 \pi^{2}} \delta_{i}^{G S}} \mid\left(W+\frac{1}{4 \pi^{2}} \delta_{i}^{G S} W_{S}\right)\right. \\
& \left.-\left.\left(T_{i}+\bar{T}_{i}\right) W_{T_{i}}\right|^{2}-3|W|^{2}\right\},
\end{aligned}
$$

where $W_{\phi}=\partial W / \partial \phi, \phi=S, T_{i}$ and $Y$ is defined in eq.(3). The simplest case occurs when $\delta_{i}^{G S}=0$. This happens for instance in the $Z_{2} \times Z_{2}$ orbifold with standard embedding [17]. From now on we will take this as our guide example since it actually gives the essential features of all the cases, but we will address in subsection 2.5 all the (slight) departures from the $\delta_{i}^{G S}=0$ results in a generic case. Notice that for $\delta_{i}^{G S}=0 \quad W^{n p}$ admits the decomposition $W^{n p}\left(S, T_{i}\right)=\Omega(S) / \prod_{i=1}^{3} \eta\left(T_{i}\right)^{2}$, even if $G$ is not simple. For the sake of 
simplicity we will work for the moment with just an overall modulus $T=T_{1}=T_{2}=T_{3}$, parametrizing the global size of the compactified space. Then the scalar potential is given by $[8]$

$$
V=\frac{|\eta(T)|^{-12}}{2 S_{R}\left(2 T_{R}\right)^{3}}\left\{\left|2 S_{R} \Omega_{S}-\Omega\right|^{2}+\left(\frac{3 T_{R}^{2}}{\pi^{2}}\left|\hat{G}_{2}\right|^{2}-3\right)|\Omega|^{2}\right\},
$$

where $\hat{G}_{2}=-\left(\frac{\pi}{T_{R}}+4 \pi \eta^{-1} \frac{\partial \eta}{\partial T}\right)$ and $S_{R} \equiv \operatorname{Re} S, T_{R} \equiv \operatorname{Re} T$. As has been noted in ref.[8], for a given reasonable value of $S$ this potential always develops a minimum in $T$ very close to the point $T=1.2$. Therefore, provided $S$ takes a reasonable value, the theory will be automatically compactified with a very sensible value of $T$. However, it is not obvious that the former will happen as will become clear shortly. The condition of stationary point in the dilaton, i.e. $\partial V / \partial S=0$, can be satisfied in two different ways:

$$
\begin{array}{ll}
\text { i) } & 2 S_{R} W_{S}-W=0 \\
\text { ii) } & 4 S_{R}^{2} \Omega_{S S}\left(2 S_{R} \Omega_{S}-\Omega\right)^{*}=E \Omega^{*}\left(2 S_{R} \Omega_{S}-\Omega\right)
\end{array}
$$

with $E=2-\frac{3 T_{R}^{2}}{\pi^{2}}\left|\hat{G}_{2}\right|^{2}$. In order to see whether $\left.i\right)$ and $\left.i i\right)$ correspond to true minima in $S$ or not it is necessary to calculate the Hessian?. Working in the general case $\Omega(S)=$ $\sum_{a} d_{a} e^{-3 k_{a} S / 2 \beta_{a}}$ it is convenient to define the parameters $x_{a}=2 S_{R}\left(3 k_{a} / 2 \beta_{a}\right)$, which for any reasonable value of $S_{R}$ and $\beta_{a}$ are much larger than 1 . For the first possibility i) it can be verified that the Hessian $H=V_{p p} V_{q q}-V_{p q}^{2}$ (with $p=\operatorname{Re} S=S_{R}, q=\operatorname{Im} S$ ) can be expressed as a sum of several terms of different order in $x_{a}$. Retaining only the dominant part we find

$$
\begin{gathered}
V_{p p}=V_{q q}=\frac{4 S_{R}}{|\eta(T)|^{12}\left(2 T_{R}\right)^{3}}\left|\Omega_{S S}\right|^{2} \\
V_{p q}=\frac{-2}{|\eta(T)|^{12}\left(2 T_{R}\right)^{3}} \operatorname{Im}\left(\Omega_{S S} \Omega_{S}^{*}\right) .
\end{gathered}
$$

So $\left|V_{p q}\right|<<\left|V_{p p}\right|$ and $H>0$. Consequently, if (13) has a solution for reasonable $S_{R}$, it will correspond to a minimum. Analogously, for the case $i i$ ), retaining again the dominant part in $x_{a}$, we find

$$
V_{p p}=-V_{q q}=\frac{-8 S_{R}^{2}}{|\eta(T)|^{12}\left(2 T_{R}\right)^{3} E} \operatorname{Re}\left(\Omega_{S S S} \Omega_{S S} e^{-i \sigma}\right)
$$

\footnotetext{
${ }^{2}$ This was performed in ref.[8] for $\Omega(S)=c+d e^{-3 S / 2 \beta}$ and $c<<1$, finding that condition (13) always corresponds to a minimum in $S$ in the weak coupling limit $\left(S_{R} \gtrsim 1\right)$.
} 


$$
V_{p q}=\frac{-8 S_{R}^{2}}{|\eta(T)|^{12}\left(2 T_{R}\right)^{3} E} \operatorname{Im}\left(\Omega_{S S S} \Omega_{S S} e^{-i \sigma}\right)
$$

where $\left(2 S_{R} \Omega_{S}-\Omega\right)=\left|2 S_{R} \Omega_{S}-\Omega\right| e^{i \sigma / 2}$. Therefore $H<0$ and we conclude that the case (14) never corresponds to a realistic minimum. We have checked these results numerically.

Hence, it is enough in the subsequent analysis to focus our attention on the solutions of type (13). It is also worth noticing that these solutions always lead to the same values of $T$ in the minimum, independently of the value of $S$, as it is easily deduced from (12,13); more precisely $T=1.23$. Of course other minima, with the same physical characteristics, appear for all the values of $T$ related to the previous one by a modular transformation (5).

\subsection{Hidden sector with a unique condensate}

This case has already been considered in the literature. Inserting simply (10) (with $\left.\delta^{G S}=0\right)$ in (12) we find a potential without minima in $S$, as it can be deduced from (13). This is schematically shown in fig.1 where $V$ is represented versus $S_{R}$ for a typical group and setting $T$ at its minimizing value. The maximum in $S_{R}$ corresponds to condition (14). Therefore, depending on the initial condition for $S_{R}$, it either runs away to infinity, thus leading to a free theory, or enters in a strong coupling regime $\left(S_{R} \rightarrow 0\right)$. Both cases are clearly unrealistic. For the general case $\left(\delta^{G S} \neq 0\right)$ the situation is exactly the same (see subsection 2.5 below).

\subsection{Hidden sector with two gaugino condensates}

The possibility of having two or more gauge group factors in the hidden sector has been previously considered in refs. $[21,6,7,12,22]$. This is in fact the most usual scenario in four dimensional strings (see e.g.[30]). However the minimization of the complete dualinvariant potential has not been performed up to now. Before starting it should be said that in string theory the rank of the hidden gauge group $G=\prod G_{a}$ is strongly restricted by the fact that its contribution to the total conformal central charge, plus that of the standard model, cannot exceed 22. Hence, $c_{\text {hidden }}=\sum_{a} c_{a}=\sum_{a} \frac{\operatorname{dim}\left(G_{a}\right) k_{a}}{k_{a}+C\left(G_{a}\right)} \leq 22-c_{S M}$, where $c_{S M}$ is the contribution of the standard model. For $k=1, c$ coincides with the rank of the corresponding group, otherwise it is higher. Consequently $\operatorname{rank}(G) \leq 18$, where the equality can only be achieved if all the groups, including the standard model one, are level 1. This restriction is crucial to imagine possible scenarios. Let us consider, to 
start with, a compactification in which the hidden sector is pure YM with gauge group $G=G_{1} \times G_{2}$, where $G_{1}$ and $G_{2}$ are simple, and $\delta^{G S}=0$. Then the corresponding condensation superpotential, see eq.(10), is

$$
W^{n p}=d_{1} \frac{e^{-3 k_{1} S / 2 \beta_{1}}}{\eta^{6}(T)}+d_{2} \frac{e^{-3 k_{2} S / 2 \beta_{2}}}{\eta^{6}(T)},
$$

where $\beta_{1}, \beta_{2}$ are the 1 -loop coefficients of the $G_{1}$ and $G_{2}$ beta functions respectively. As we know from section 2.1 any realistic minimum of the potential $V(S, T)$, see eq.(12), arising from (19) should satisfy the condition (13), which in this case reads:

$$
\begin{aligned}
2 S_{R} W_{S}-W=0 \quad & \rightarrow \quad S_{R}=\frac{2}{3\left(k_{1} \beta_{1}^{-1}-k_{2} \beta_{2}^{-1}\right)} \log \frac{d_{1}\left(3 S_{R} k_{1} \beta_{1}^{-1}+1\right)}{d_{2}\left(3 S_{R} k_{2} \beta_{2}^{-1}+1\right)} \\
& \rightarrow \quad \operatorname{Im} S=\frac{2 \pi(2 n+1)}{3\left(k_{1} \beta_{1}^{-1}-k_{2} \beta_{2}^{-1}\right)}, n \in Z
\end{aligned}
$$

Clearly the value of $S$ is not related to the specific $T$-dependence of $W$. Notice that $\operatorname{Im} S$ is simply such that the two condensates get opposite signs giving a cancellation between them. If $S_{R}$ is realistic, i.e. $S_{R}=O(1)$, then $3 k_{a} S_{R} \beta_{a}^{-1}>>1$ and condition (20) becomes analogous to

$$
W_{S}=0 \quad \rightarrow \quad S_{R}=\frac{2}{3\left(k_{1} \beta_{1}^{-1}-k_{2} \beta_{2}^{-1}\right)} \log \frac{d_{1} \beta_{2} k_{1}}{d_{2} \beta_{1} k_{2}}
$$

The condition $W_{S}=0$ was used in refs.[7,12] to look for realistic scenarios. We see here that it can be properly justified. Clearly the value of $S_{R}$ is related to the value of the $d_{a}$ coefficients. Using the field theory value $d_{a}=-\beta_{a} / 6 e$, see eq.(10), it can be easily verified that eq.(20) cannot be fulfilled for positive $S_{R}$ and $k_{1}=k_{2}$. For $k_{1} \neq k_{2}$ it is possible to find non-trivial solutions to eqs.(20,21), but they are always far from the realistic range, i.e. $S_{R}<<1$. E.g. for $S U(3)_{k_{1}=1} \times S U(4)_{k_{2}=2}$ and $S U(6)_{k_{1}=1} \times S U(7)_{k_{2}=2}$ there appear minima at $S_{R}=0.0447$ and $S_{R}=0.0535$ respectively. These results cannot be essentially improved for other choices of the gauge group. Certainly $T$ becomes fixed at a reasonable value, but the fact that the gauge coupling constant enters in the strong coupling regime does not allow this result to be taken very seriously.

It has been argued in ref.[6] that a possible way out of this problem is the appearance of "stringy" field-independent threshold corrections $\Delta_{a}$ to be added to eq.(1). Then the value of $d$ in eq.(10) is modified in a different way for each condensate

$$
d_{a} \quad \rightarrow \quad d_{a}^{\prime}=d_{a} e^{-3 \Delta_{a} / 2 \beta_{a}}
$$


Depending on the size of these corrections, it might occur that eq. (20) has a reasonable solution for some choice of $G_{1}$ and $G_{2}$. On the other hand, these contributions have been calculated in ref.[16] in the context of the $Z_{3}$ orbifold and in ref.[31] for fermionic constructions associated with $Z_{N} \times Z_{M}$ compactifications. In all cases they are very small, far from fixing up the situation. So we conclude that this possibility is rather speculative, a reasonable choice being to take $\Delta_{a}=0$, as we will do throughout. In any case all the results of the paper are straightforwardly modified using eq.(23) to count the effect of these field-independent contributions.

\subsection{Hidden sector with three or more condensates}

The previous results are not improved when more than two condensates are considered. In general, if the hidden sector gauge group contains $n$ factors $G=\prod_{a=1, \ldots, n} G_{a}$, then

$$
W^{n p}=\sum_{a=1, \ldots, n} d_{a} \frac{e^{-3 k_{a} S / 2 \beta_{a}}}{\eta^{6}(T)}
$$

with the same notation as in eq.(19). As was shown in subsection 2.1 condition (13) is the only one that can lead to realistic minima. This implies

$$
\sum_{a=1}^{n} z_{a}=0
$$

where $z_{a}=d_{a}\left(1+3 S_{R} k_{a} \beta_{a}^{-1}\right) e^{-3 k_{a} S / 2 \beta_{a}}$. For $n=3$, i.e. in the case of three condensates, (25) leads to $\left|z_{a}+z_{b}\right|=\left|z_{c}\right|(a \neq b \neq c \neq a)$ which, denoting $z_{a}=\left|z_{a}\right| e^{i \varphi_{a}}$, reads

$$
\cos \left(\varphi_{a}-\varphi_{b}\right)=\frac{\left|z_{c}\right|^{2}-\left|z_{a}\right|^{2}-\left|z_{b}\right|^{2}}{2\left|z_{a}\right|\left|z_{b}\right|}, \quad a \neq b \neq c \neq a .
$$

Now, using $-1 \leq \cos \left(\varphi_{a}-\varphi_{b}\right) \leq 1$, we obtain bounds on $S_{R}$ which turn out to be surprisingly strong. In fact they virtually exclude any possibility of finding realistic minima with three condensates, as is shown in table 1. (Actually it is even difficult to find examples with true minima, as the numerical analysis reveals.) These results are basically maintained for $n>3$. In ref.[22] a stationary point was found for the rather bizarre case $G=S U(2)_{k=1} \times S U(2)_{k=2}^{4} \times S U(2)_{k=3}^{4}$, where $k$ denotes the levels of the associated Kac-Moody algebras. We have found that this stationary point really corresponds to a minimum, but it occurs at $S_{R}=0.017$, again a completely unrealistic value. In addition this solution does not break supersymmetry. 


\subsection{The $\delta^{G S} \neq 0$ case}

The general case occurs when $\delta_{i}^{G S} \neq 0$. This is reflected in eqs.(13,10) and in the resulting potential $V$, given by eq.(11). The complexity of the latter does not allow for an analytical study as that performed for $\delta_{i}^{G S}=0$. In particular, conditions (13, 14) now become much more involved. However, the numerical analysis shows that $V$ essentially exhibits the same features as the one for the $\delta_{i}^{G S}=0$ case, see eq.(12). Namely, for a unique condensate there is no minimum at all in $S$, while for two or more condensates examples can be found with minima but for unrealistic values of $Y=2 / g_{\text {string }}^{2}$. In any case, it is interesting to notice that it may be impossible for the last scenario (i.e. two or more pure YM sectors) to occur in many instances, as modular invariance arguments indicate. To see this consider first the $Z_{3}$ and $Z_{7}$ cases. These orbifolds do not possess $N=2$ subsectors, which implies that the coefficient of $\log \eta\left(T_{i}\right)$ in eq.(1) must vanish [16,17], i.e. $b_{i}^{\prime a}=16 \pi^{2} \beta^{a} / 3=2 k^{a} \delta_{i}^{G S}$. Taking into account that $\delta_{i}^{G S}$ are universal for all the gauge group factors it is clear that this cancellation cannot take place simultaneously for two sectors with different $\left(k^{-1} \beta\right)$ coefficients. Alternatively, notice that, since for these orbifolds $\eta(T)$ does not appear in the expression of the condensation superpotential (10), there is no modular transformation of $S$ (see eq.(7)) able to give the correct modular weight -3 to a sum of pure YM condensates $W^{n p} \propto \sum_{a} e^{-3 k_{a} S / 2 \beta_{a}}$ unless all the $\left(k_{a}^{-1} \beta_{a}\right)$ factors coincide, which for practical purposes is equivalent to having a unique condensate. This argument can be extended to the rest of the $Z_{N}$ orbifolds since all of them have planes which are never completely rotated.

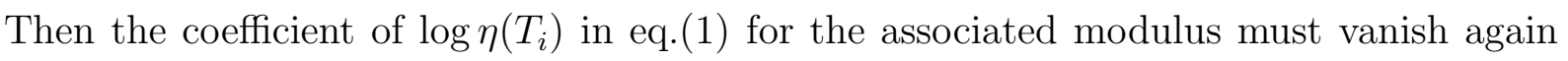
$[16,17]$, leading to the same conclusion as for the $Z_{3}$ and $Z_{7}$ orbifolds. This rule is actually satisfied by all the pure YM examples constructed up to now, e.g. the above mentioned $S U(3) \times S U(3) Z_{4}$ model of ref.[29].

On the contrary, there is nothing in principle against having multiple pure YM gaugino condensation in $Z_{N} \times Z_{M}$ orbifolds.

The results of this section show that a scenario with a pure YM hidden sector is not viable from the phenomenological point of view. The most interesting situation takes place when there are two or more condensates, which, apparently, is only possible for $Z_{N} \times Z_{M}$ orbifolds. However, the minima of the scalar potential found in this case are clearly unrealistic, since they have $S_{R}<<1$. This leads us to consider in the next section the most general (and frequent) case, namely gaugino condensation in the presence of matter. 


\section{Gaugino condensation in the presence of matter}

\subsection{General procedure and the $\delta^{G S}=0$ case}

It is known that the existence of hidden matter is the most general situation and occurs in all promising string constructions [25,32]. For orbifold compactifications, explicit condensing examples can be found. E.g. in ref.[25] phenomenologically interesting $Z_{3}$ models with $G=S U(3) \times S U(2) \times U(1)_{Y} \times[S O(10)]^{\prime}$ and three 16 's hidden matter representations were constructed. In ref.[33] two $Z_{7}$ models with $S U(3) \times S U(2) \times U(1)^{5}$ observable gauge group and $S O(10), S U(5) \times S U(3)$ hidden sectors with hidden matter $10,3(5+\overline{5})+7(3+\overline{3})$ respectively were constructed. The rules for building up $\left[S U(3) \times S U(2) \times U(1)^{5}\right] \times[G]^{\prime}$ models in the framework of $Z_{3}$ and $Z_{7}$ orbifolds are given in [34,33]. Examples in the framework of other $Z_{N}$ orbifolds, e.g. a $Z_{4}$ model with $E_{6} \times S U(2)$ observable sector and $E_{7}$ hidden gauge group plus 2(56) hidden matter representations, can be found in [30,35]. Many more examples can be constructed.

Gaugino condensation in the presence of massive matter fields was studied several years ago for globally supersymmetric field theories [36-38]. However, there is at present no generally accepted formalism describing the condensation in the presence of massless matter (see refs.[37,38]) and it should be noticed that, strictly speaking, in the context of superstring theories there are no light matter fields with mass terms in the $D=4$ effective Lagrangian. Fortunately, these fields usually have trilinear couplings between them (see eq.(4)) and, therefore, one can proceed as if they were massive with a dynamical mass given by the VEV of another matter field. Furthermore one has to take into account that the effective field theory is now locally supersymmetric. This has been done in the past year in refs. $[11,12,14]$. We will just write here the final result. In the case of $G=S U(N)$ with $M(N+\bar{N})$ " quark" representations $Q_{\alpha}, \bar{Q}_{\alpha}, \alpha=1, \cdots, M$, coupled to a set of singlet fields $\left\{A_{r}\right\}$ in the usual way (see eq.(四)

$$
W^{t r}=\sum_{r, \alpha, \beta} h_{r \alpha \beta}\left(T_{i}\right) A_{r} Q_{\alpha} \bar{Q}_{\beta}
$$

(of course many Yukawa couplings $h_{r \alpha \beta}$ can be zero), the complete condensation superpotential can be written as

$$
W^{n p}(S, T, A)=-N\left(32 \pi^{2} e\right)^{\frac{M}{N}-1}[\operatorname{det} \mathcal{M}]^{\frac{1}{N}} \frac{e^{-\frac{8 \pi^{2} k}{N} S}}{\prod_{i=1}^{3}\left[\eta\left(T_{i}\right)\right]^{\frac{2}{N}\left(b_{i}^{\prime}-2 k \delta_{i}^{G S}\right)}},
$$

where $\mathcal{M}_{\alpha \beta}=\sum_{r} h_{r \alpha \beta}\left(T_{i}\right) A_{r}$. In this result the trilinear piece $W^{t r}$ (eq.(27)) has also

been incorporated. It can be checked that (28) has the correct modular weight -3 . 
Notice that the exponent in the numerator is simply $-3 k S / 2 \beta$ where $\beta$ corresponds to the pure YM $S U(N)$ theory. $W^{n p}$ in eq.(28) is for quark masses $\left(\sim h\left(T_{i}\right) A\right)$ smaller than the condensation scale $\Lambda$. In the opposite case the result is exactly the same but with a slightly different prefactor. The non-vanishing Yukawa couplings $h_{r \alpha \beta}$ are simply 1 for untwisted or mixed couplings while they have a non-trivial dependence on $T_{i}$ for the twisted case [26,27], see eq.(化). These formulae are easily extended to the case of a non-simple gauge group $G=\prod_{a} G_{a}$.

Of course, for this approach to be consistent, the VEVs of the $A$ fields must become at the end different from zero as a consequence of the minimization of the potential, otherwise the "quark" fields are not massive. However, this has not been studied in the literature yet, and it is not obvious to happen. As in the previous section we take the $\delta_{i}^{G S}=0$ case as a useful guide example (which is known to occur in the $Z_{2} \times Z_{2}$ orbifold with standard embedding [17]) and in the next subsection we will extend the results to the general case. We also work for convenience with an overall modulus $T$, a generic $A$ field of the untwisted type (so $\operatorname{det} \mathcal{M}=A^{M}$ ) and untwisted $Q$ fields?. This simplified approach gives the correct results when the number of quarks to which $A_{i}$ ( $i$ denotes a holomorphic index) gives mass in (27), say $M_{i}$, is the same in the three complex planes (i.e. $M_{1}=M_{2}=M_{3}=\frac{1}{3} M$ ). Then the minimization equations allow $<A_{1}>=<A_{2}>=<A_{3}>\equiv<A>,<T_{1}>=<T_{2}>=<T_{3}>\equiv<T>$. Under more general circumstances this approach gives an average of $\left\langle A_{i}\right\rangle,\left\langle T_{i}\right\rangle$. The Kähler potential for untwisted fields and $\delta_{i}^{G S}=0$ is very well known [39]

$$
K_{1-\text { loop }}=-\log (S+\bar{S})-3 \log \left[(T+\bar{T})-|A|^{2}\right] .
$$

The corresponding modular invariant potential is thus

$$
\begin{aligned}
V= & \frac{1}{2 S_{R}\left(2 T_{R}-|A|^{2}\right)^{3}}\left\{\left|2 S_{R} W_{S}-W\right|^{2}+\frac{1}{3}\left(2 T_{R}-|A|^{2}\right)\left|\frac{\partial W}{\partial A}+\bar{A} \frac{\partial W}{\partial T}\right|^{2}\right. \\
& \left.+\frac{1}{3}\left(2 T_{R}-|A|^{2}\right)^{2}\left|\frac{\partial W}{\partial T}-\frac{3 W}{2 T_{R}-|A|^{2}}\right|^{2}-3|W|^{2}\right\} .
\end{aligned}
$$

For $|A|^{2}=2 T_{R}$ there exists an infinite barrier which allows us to restrict the values of $|A|^{2}$ to the range $|A|^{2}<2 T_{R}$. This is also the condition for kinetic energy positivity. Substituting (28) in (30) for one or more condensates corresponding to a group $G=$ $\prod_{a=1}^{n} G_{a}$, one finds a quite involved expression for $V(S, T, A)$ which requires a numerical

\footnotetext{
${ }^{3}$ It is worth noticing here that, concerning modular transformations and Yukawa couplings, most of the matter fields in $Z_{N} \times Z_{M}$ orbifolds "behave" as untwisted ones. For $Z_{N}$ examples with all the hidden matter in the untwisted sector see e.g. refs.[30,35].
} 
analysis. This analysis indicates that $V$ does not have true minima for $n<3$. This behaviour can be intuitively understood in the following way. For a realistic case $\left(S_{R}\right.$, $\left.T_{R}=O(1)\right)$ one expects $<|A|^{2}><<S_{R}, T_{R}$, since $A$ is expected to have a vanishing VEV at the perturbative level. At least this certainly should happen for $|A|<\Lambda$, which, as will become clear shortly, is the relevant case for us. Then, since $W^{n p}$ in (28) is a sum of terms that are power-law functions of $A$, it is clear that the term $\propto|\partial W / \partial A|^{2}$ in (30) dominates the potential (except within a very narrow band around $\partial W / \partial A=0$ ). Hence, we can approximate

$$
V \sim \frac{1}{6 S_{R}\left(2 T_{R}\right)^{2}}\left|\frac{\partial W}{\partial A}\right|^{2}
$$

which has the absolute minimum at

$$
\partial W / \partial A=0
$$

and possible relative minima at $\partial^{2} W / \partial A^{2}=0$ (note that the requirement (32) leaves global SUSY unbroken in the weak coupling limit). No one of these conditions can be nontrivially fulfilled with the superpotential of eq.(28) when a single condensate is considered. Therefore $V(S, T, A)$ does not have minima. These heuristic results are fully confirmed by the numerical analysis. When several condensates are involved the situation is more complex and is studied in subsection 3.3, where it is shown that only for three or more condensates and under particular circumstances there can emerge interesting solutions.

The situation expounded above might seem phenomenologically discouraging. Fortunately this is not so, thanks to the crucial role played by the perturbative superpotential. The importance of the interplay between the perturbative and non-perturbative parts of the superpotential for a good supersymmetry breaking has already been pointed out in refs.[40,7] and, more recently, in ref.[13]. The point is that, besides the coupling to the quarks, $A$ has perturbative interactions with other singlet fields. This changes the situation dramatically. To see this, consider the following simplified ansatz for $W$

$$
\begin{aligned}
W & =W^{n p}+W^{\text {pert }} \\
W^{\text {pert }} & =A^{3}
\end{aligned}
$$

(recall that $A$ is assumed to be untwisted). Now, it is easy to check that condition (32) has a non-trivial solution. For the case of a unique condensate eq. (32) leads to

$$
A^{3}=\left(\frac{M}{3}\right)^{\frac{3 N}{3 N-M}}\left(32 \pi^{2} e\right)^{\frac{3(M-N)}{3 N-M}} \frac{e^{-24 \pi^{2} k S /(3 N-M)}}{\eta(T)^{6}},
$$

\footnotetext{
${ }^{4}$ We thank J. Louis for putting forward this possibility to us.
} 
where we have used $\sum_{i} b_{i}^{\prime}=3 N-M$ in the untwisted case. Note that certainly if $S_{R}, T_{R}=O(1)$ then $<|A|^{2}><<S_{R}, T_{R}$, as is required for consistency. Furthermore $A^{3}=\frac{M}{96 \pi^{2}} U$, where $U$ is the gaugino composite bilinear field (see section 2), which indicates that $|A|<\Lambda$ is indeed the relevant case for us. Moreover, the F-term associated with $A$ $\left(\propto\left[W_{A}+W K_{A}+\bar{A}\left(W_{T}+W K_{T}\right)\right]\right)$ is clearly dominated by $W_{A}$. Hence, we can substitute (35) in $W$, thus obtaining an effective superpotential that depends on $S$ and $T$ only

$$
\begin{gathered}
W^{e f f}=\tilde{d} \frac{e^{-3 k S / 2 \tilde{\beta}}}{\eta^{6}(T)}, \\
\tilde{\beta}=\frac{3 N-M}{16 \pi^{2}}, \quad \tilde{d}=\left(\frac{M}{3}-N\right)\left(32 \pi^{2} e\right)^{\frac{3(M-N)}{3 N-M}}\left(\frac{M}{3}\right)^{\frac{M}{3 N-M}},
\end{gathered}
$$

where $\tilde{\beta}$ is the beta function of the complete $S U(N)$ theory with quarks. Again, the numerical analysis confirms the validity of this procedure for "integrating out" the $A$ field. Notice that $W^{e f f}$ still has the correct modular weight -3 , exhibiting the same form as in the pure YM case甲, eq. (10), except for the different values of the exponent $(-3 k / 2 \tilde{\beta})$ and the prefactor $\tilde{d}$ (this modification will become crucial). In consequence, the form of the scalar potential of eq.(12) and the subsequent analysis hold for this case. In particular, we can assure that, even in the presence of matter, the potential for a unique condensate does not develop true minima. Things change dramatically, however, for multiple gaugino condensation. The case of two condensates is especially interesting. Then

$$
W^{e f f}=\tilde{d}_{1} \frac{e^{-3 k_{1} S / 2 \tilde{\beta}_{1}}}{\eta^{6}(T)}+\tilde{d}_{2} \frac{e^{-3 k_{2} S / 2 \tilde{\beta}_{2}}}{\eta^{6}(T)} .
$$

As was shown in subsection 2.3 the associated potential can only have a realistic minimum at

$$
\begin{aligned}
2 S_{R} W_{S}^{\text {eff }}-W^{e f f}=0 & \rightarrow \quad S_{R}=\frac{2}{3\left(k_{1} \tilde{\beta}_{1}^{-1}-k_{2} \tilde{\beta}_{2}^{-1}\right)} \log \frac{\tilde{d}_{1}\left(3 S_{R} k_{1} \tilde{\beta}_{1}^{-1}+1\right)}{\tilde{d}_{2}\left(3 S_{R} k_{2} \tilde{\beta}_{2}^{-1}+1\right)} \\
& \rightarrow \quad \operatorname{Im} S=\frac{2 \pi(2 n+1)}{3\left(k_{1} \tilde{\beta}_{1}^{-1}-k_{2} \tilde{\beta}_{2}^{-1}\right)}, n \in Z
\end{aligned}
$$

(see eqs. 20, 21)). Recall here that this condition is equivalent to $W_{S}^{\text {eff }}=0$ for $S_{R}=O(1)$, see eq.(22). The crucial difference with respect to the pure YM case comes from the values of $\tilde{\beta}_{i}, \tilde{d}_{i}$. These depend only on which the gauge groups and matter contents

\footnotetext{
${ }^{5}$ This was first noticed by Kaplunovsky and Louis $[41,13]$.

${ }^{6} \mathrm{We}$ are supposing here for simplicity that the $A$-field giving mass to the quarks of the first gauge group is different from that of the other.
} 
of the hidden sector are. It will be seen shortly that there are many possibilities for these leading to a value of $S_{R}\left(=g_{\text {string }}^{-2}\right)$ in the realistic range $\left(S_{R} \sim 2\right)$. In this way, there naturally arises a wide and very interesting class of scenarios for which a good perturbative unification is plausible. For the sake of definiteness we have represented in table 2 the complete set of $S U\left(N_{1}\right) \times S U\left(N_{2}\right)$ scenarios with $k_{1}=k_{2}=1, N_{a}>M_{a}$ and $N_{1} \neq N_{2}$ for which $1 \leq S_{R} \leq 3.5$ (for $N_{1}=N_{2}$ there are many more possibilities, as is commented below). We have allowed for this rather wide range because the gauge coupling constant $g_{g u t}^{2}$ at the unification mass $\left(M_{g u t} \sim 10^{16} \mathrm{GeV}\right)$ is not exactly $S_{R}^{-1}$ but it is corrected by threshold contributions $[16,17]$ and the running between $M_{\text {string }}$ and $M_{\text {gut }}$ (see ref.[42]). Both facts are model-dependent. We are also assuming that the standard model gauge group has $k=1$, otherwise the realistic range for $S_{R}$ would be $1 \leq k S_{R} \leq 3.5$, thus leading to additional classes of viable scenarios. Notice however that for $k \neq 1$ the range of the hidden gauge group is restricted further. These results are in impressive agreement with those obtained numerically by using the complete potential of eq.(30) and the superpotential of eqs.(33, 34). As in the pure YM case the value of $T$ in the minimum is always $T=1.23$ or any other value obtained from this by a modular transformation (5), so duality is spontaneously broken as it was noted in refs.[8,9]. Notice that the values of $S$ and $T$ are not related to each other, i.e. the solution of $\partial V / \partial S=0$ does not depend on the specific dependence of $K$ and $W$ on $T$ and vice versa. We have represented the potential for a typical case in fig.2, where duality invariance can be clearly recognized.

To understand intuitively these results it is convenient to recall that conditions (39), (40) for $S_{R}=O(1)$ are almost equivalent to $\partial W^{\text {eff }} / \partial S=0$ (see eq.(22)). If we make some further approximations, neglecting small logarithms, we obtain a useful formula for $S_{R}$

$$
S_{R} \simeq 0.17 \frac{N_{2} M_{1}-N_{1} M_{2}}{k_{1}\left(3 N_{2}-M_{2}\right)-k_{2}\left(3 N_{1}-M_{1}\right)},
$$

corresponding to a gauge group $S U\left(N_{1}\right) \times S U\left(N_{2}\right)$ with $M_{1}\left(N_{1}+\bar{N}_{1}\right)$ and $M_{2}\left(N_{2}+\bar{N}_{2}\right)$ quark representations. Clearly $S_{R}$ can be $O(1)$ for many combinations of $N_{1}, N_{2}, M_{1}, M_{2}$, as is illustrated in table 2 , so we see that the requirement of a reasonable gauge coupling constant does not imply fine-tuning. In particular, it is clear from (41) that for a hidden gauge group $S U(N)_{k=1} \times S U(N)_{k=1}$, provided $M_{1} \neq M_{2}$, the value of $S_{R}$ is essentially independent from the matter content $\left(S_{R} \sim 0.17 N\right)$ and quite reasonable for $6 \leq N \leq 10$ (notice here that $S U(10)_{k=1} \times S U(10)_{k=1}$ has the maximum rank allowed). This fact is illustrated at the end of table 2 with a few examples out of the 145 existing ones. It is worth noticing that examples with $N \leq M<3 N$ can also be found (some of them are shown in table 3). This seems to be a departure from previous results in the context 
of global supersymmetry. This is in fact a polemical point in the literature. While it is claimed in ref.[37] that for $N \leq M$ no effective superpotential describing gaugino condensation can be formulated since the "squark" condensate $\Pi=\operatorname{det}\left(\bar{Q}_{i} Q_{j}\right)$ vanishes identically, in ref.[38] it is argued that this condensate can be a different expression of the squark fields, thus permitting normal gaugino condensation. In this second case the scenarios found with $N \leq M$ are on the same footing as the others. If we allow for $k_{1} \neq k_{2}$ many additional scenarios appear, as is reflected in table 4. Finally, let us emphasize the fact that all the minima found previously do correspond to a weak coupling regime, which makes these solutions very reliable.

Once we have seen that it is possible to fix the dilaton satisfactorily in a wide class of scenarios, let us examine the supersymmetry breaking issue. Actually, in the previously determined minima supersymmetry is spontaneously broken due to the non-vanishing of the F-term associated with $T$. Notice that the $S \mathrm{~F}$-term is zero due to the minimum condition (39,40). On the other hand, the cosmological constant $\Lambda_{\text {cosm }}$ is generically different from zero and negative, so we have to assume the existence of some unknown contributions rendering $\Lambda_{\text {cosm }}=0$. The supersymmetry breaking scale is essentially given by the gravitino mass $m_{3 / 2}$, which for the scenarios considered above (i.e. two condensing groups in the hidden sector) is

$$
m_{3 / 2}=e^{K / 2}\left|W^{e f f}\right| \simeq \frac{1}{\left(2 S_{R}\right)^{1 / 2}\left(2 T_{R}\right)^{3 / 2}|\eta(T)|^{6}}\left|\tilde{d}_{1}\left(1-\frac{k_{1} \tilde{\beta}_{2}}{k_{2} \tilde{\beta}_{1}}\right)\right| e^{-3 k_{1} S_{R} / 2 \tilde{\beta}_{1}}
$$

where $W^{\text {eff }}$ is given by (38) and we have used $W_{S}^{e f f}=0$ which is a condition practically equivalent to (39) for realistic $S_{R}$. The requirement of having $10^{2} \mathrm{GeV} \leq m_{3 / 2} \leq 10^{4} \mathrm{GeV}$ (which is necessary in order to preserve the virtues of supersymmetry to solve the hierarchy problem) restricts the viable possibilities of the hidden sector further. There survive, however, many of them. In particular, for $k_{1}=k_{2}=1$ there are 527 viable scenarios. Eleven of them have $N_{a}>M_{a}$ (\#6, 9, 12,14,17, 19, 28, 32, 35, 36, 37 in table 2). The rest have $M \geq N$ in one of the two sectors (80 of them are shown in table 3 ). Clearly, there is still no need of fine-tuning in the hidden sector to arrange realistic values for $g_{\text {string }}$ and $m_{3 / 2}$ simultaneously. All this all reflects the capacity of gaugino condensation as the source of a hierarchical supersymmetry breakdown. To understand the numeric results intuitively notice that the most important factor in (42) is the exponential. Using the approximate value of $S_{R}$ given in eq.(41) and doing some further approximations it can be seen that a realistic value for $m_{3 / 2}$ requires

$$
M_{2}-M_{1} \simeq 6\left(N_{2}-N_{1}\right)
$$


This empirical formula is well satisfied by the 527 above mentioned scenarios. A very remarkable fact is that once we require a reasonable value for $m_{3 / 2}$ the corresponding value of $S_{R}$ is automatically placed about $S_{R}=2$, i.e. the optimum value (see tables 2 , $3)$. Therefore, good values for $g_{\text {string }}^{-2}$ and $m_{3 / 2}$, far from being incompatible, are correlated! Actually this pleasing and non-trivial result is a characteristic of level 1 constructions, which seem to be favoured from this point of view. E.g. for $k_{1}=1, k_{2}=2$ there are 76 examples with $S_{R}$ and $m_{3 / 2}$ inside reasonable ranges ( 5 of them with $N>M$ ), to be compared with the 527 examples for $k_{1}=k_{2}=1$. They are displayed in table 4 , from which it is clear that the typical value of $S_{R}$ is located about $S_{R}=1$.

Let us finally see that, as usual, soft breaking masses for the scalars similar to the gravitino mass are generated. The dependence of the Kähler potential and the scalar potential on an untwisted field $Q$ is the same as that on $A$ in eqs.(29,30). Consequently, using eq.(32) and $W_{S}^{e f f} \simeq 0$ (see eqs.(39,40), the physical mass $m_{Q}$ generated for the canonically normalized scalar component of $Q$ can be written, after some algebra, as

$$
m_{Q}^{2}=m_{3 / 2}^{2}-e^{K}\left|\frac{1}{3}\left(2 T_{R}-|A|^{2}\right) \frac{\partial W}{\partial T}-W\right|^{2}+V_{o}+\ldots
$$

where $m_{3 / 2}^{2}=e^{K}|W|^{2}, V_{o}$ is essentially the cosmological constant (which we are assuming to be zero) and the dots stand for other terms involving $A$, which are negligible once one takes into account the fact that $|A|^{2}<<S_{R}, T_{R}$, see eq.(35). Substituting now the explicit form for $W$ of eq.(38) we find

$$
m_{Q}^{2} \simeq\left(1-\left(\frac{T_{R}}{\pi}\right)^{2}\left|\hat{G}_{2}(T)\right|^{2}\right) m_{3 / 2}^{2} \simeq\left(0.81 m_{3 / 2}\right)^{2}
$$

where $\hat{G}_{2}(T)$ is defined in eq.(12) and we have used the value of $T$ in the minimum, i.e. $T=1.23$. Notice that $m_{Q}$ is modular invariant.

\subsection{The general case}

The results so far obtained in this section can be extended to the $\delta_{i}^{G S} \neq 0$ case with some modifications. Working still with hidden matter of the untwisted type (recall that this is the general situation in many $Z_{N}$ and $Z_{N} \times Z_{M}$ constructions) we can proceed as in the previous subsection in order to eliminate the $A$ field through condition】 (32), which in

\footnotetext{
${ }^{7}$ To reproduce step by step the arguments of subsection 3.1 it is necessary to use the Kähler potential $K$ for untwisted fields and $\delta^{G S} \neq 0$, which is not very well known although modular invariance imposes strong restrictions on it. Using the very plausible form for $K$ conjectured in ref.[14], it is easy to check that eq. (32) still holds for $\delta^{G S} \neq 0$.
} 
this case reads

$$
A^{3}=\left(\frac{M}{3}\right)^{\frac{3 N}{3 N-M}}\left(32 \pi^{2} e\right)^{\frac{3(M-N)}{3 N-M}} \frac{e^{-24 \pi^{2} k S /(3 N-M)}}{[\eta(T)]^{6-\left(12 k \delta^{G S} /(3 N-M)\right)}},
$$

where $\delta^{G S} \equiv \sum_{i} \delta_{i}^{G S}$. Again, substituting (46) in $W=W^{n p}+W^{p e r t}$ gives a superpotential which depends on $S$ and $T$ only

$$
W^{e f f}=\tilde{d} \frac{e^{-3 k S / 2 \tilde{\beta}}}{[\eta(T)]^{6-\left(3 k \delta^{G S} / 4 \pi^{2} \tilde{\beta}\right)}},
$$

where $\tilde{d}, \tilde{\beta}$ are defined in eq.(37). Note again that $W^{\text {eff }}$ has the correct modular weight -3 and a form very similar to that of the pure YM case (10). Using the Kähler potential (3), written for an overall modulus $T$, the scalar potential for a generic $W(S, T)$ is

$$
V=\frac{1}{Y\left(2 T_{R}\right)^{3}}\left\{\left|Y W_{S}-W\right|^{2}+\frac{Y}{3 Y+\frac{1}{4 \pi^{2}} \delta^{G S}}\left|3 W+\frac{1}{4 \pi^{2}} \delta^{G S} W_{S}-2 T_{R} W_{T}\right|^{2}-3|W|^{2}\right\}
$$

which is the same as (11) when $\delta_{1}^{G S}=\delta_{2}^{G S}=\delta_{3}^{G S}=\frac{1}{3} \delta^{G S}$. If this is not so, there is a certain asymmetry between $T_{1}, T_{2}$ and $T_{3}$. Then the results obtained from (48) for $T$ can be considered as an average of $T_{i}$. In any case we will see shortly that the effect of $\delta^{G S} \neq 0$ is normally small.

As was mentioned in subsection 2.5 the complexity of (48) requires the various scenarios to be analyzed numerically. It is remarkable however that the results of this analysis show that, whenever $S_{R}$ lies in the realistic range $\left(S_{R} \sim O(1)\right)$, the actual value of $S_{R}$ is still extremely close to that obtained from condition (13). In particular, for a unique condensate there is no minimum at all in $S_{R}$ and the shape of the potential is as in fig.1. For two condensates

$$
W^{e f f}=\tilde{d}_{1} \frac{e^{-3 k_{1} S / 2 \tilde{\beta}_{1}}}{[\eta(T)]^{6-\left(3 k_{1} \delta^{G S} / 4 \pi^{2} \tilde{\beta}_{1}\right)}}+\tilde{d}_{2} \frac{e^{-3 k_{2} S / 2 \tilde{\beta}_{2}}}{[\eta(T)]^{6-\left(3 k_{2} \delta^{G S} / 4 \pi^{2} \tilde{\beta}_{2}\right)}}
$$

condition (13) can be fulfilled and there appear lots of interesting scenarios, as in the $\delta^{G S}=0$ case. Therefore the corresponding value of $S$ is close to

$$
\begin{aligned}
S_{R} & =S_{R}^{\left(\delta^{G S}=0\right)}+\frac{\delta^{G S}}{4 \pi^{2}} \log |\eta(T)|^{2} \\
& \simeq \frac{2}{3\left(k_{1} \tilde{\beta}_{1}^{-1}-k_{2} \tilde{\beta}_{2}^{-1}\right)} \log \frac{\tilde{d}_{1} k_{1} \tilde{\beta}_{1}^{-1}}{\tilde{d}_{2} k_{2} \tilde{\beta}_{2}^{-1}}-\frac{0.645}{4 \pi^{2}} \delta^{G S} \\
\operatorname{Im} S & =\operatorname{Im} S^{\left(\delta^{G S}=0\right)}+\frac{\delta^{G S}}{2 \pi^{2}} \operatorname{phase}(\eta(T))
\end{aligned}
$$


where $S^{\left(\delta^{G S}=0\right)}$ is the value of $S$ for the $\delta^{G S}=0$ case, as given by eqs. 39,40). In writing (50) we have used the fact that $3 S_{R} k_{a} \beta_{a}^{-1}>>1$ (since $S_{R} \sim O(1)$ for viable scenarios) and the empirical result that the value of $T$ in the minimum obtained numerically continues being $T \simeq 1.23$ in all cases (the discrepancies only appear at the fourth significant figure). Consequently

$$
\frac{Y}{2}=g_{\text {string }}^{-2}=S_{R}+\frac{1}{8 \pi^{2}} \delta^{G S} \log \left(2 T_{R}\right) \simeq \frac{2}{3\left(k_{1} \tilde{\beta}_{1}^{-1}-k_{2} \tilde{\beta}_{2}^{-1}\right)} \log \frac{\tilde{d}_{1} k_{1} \tilde{\beta}_{1}^{-1}}{\tilde{d}_{2} k_{2} \tilde{\beta}_{2}^{-1}}-\frac{0.195}{4 \pi^{2}} \delta^{G S} .
$$

Comparing with (39), (40) it is clear that if $\delta^{G S}$ is not large (i.e. $\frac{1}{4 \pi^{2}} \delta^{G S} \lesssim 1$, as it actually happens in all the calculations of $\delta^{G S}$ performed up to now [17]) the results will not differ basically from those for $\delta^{G S}=0$. This fact is illustrated in table 5 with a few examples and some typical values of $\delta^{G S}$. We have also included the result for $\delta^{G S}=0$ to facilitate the comparison. The shape of the potential for a particular example is given in fig.3, to be compared with the corresponding one for $\delta^{G S}=0$, fig.2. On the other hand the gravitino mass for $\delta^{G S} \neq 0$ is given by

$$
\begin{aligned}
m_{3 / 2}=e^{K / 2}\left|W^{e f f}\right| & \simeq \frac{1}{Y^{1 / 2}\left(2 T_{R}\right)^{3 / 2}|\eta(T)|^{6-\left[12 \delta^{G S} /(3 N-M)\right]}}\left|\tilde{d}_{1}\left(1-\frac{k_{1} \tilde{\beta}_{2}}{k_{2} \tilde{\beta}_{1}}\right)\right| e^{-3 k_{1} S_{R} / 2 \tilde{\beta}_{1}} \\
& \simeq\left(\frac{2 S_{R}^{\left(\delta^{G S}=0\right)}}{Y}\right)^{1 / 2} m_{3 / 2}^{\left(\delta^{G S}=0\right)}
\end{aligned}
$$

where $m_{3 / 2}^{\left(\delta^{G S}=0\right)}$ is given in eq.(42) and we have used the first equation of (50), thanks to which the dependence of $m_{3 / 2}$ on $\delta^{G S}$ has almost been cancelled (there only remains a slight dependence through the value of $Y$ ). This remarkable stability of $m_{3 / 2}$ with respect to variations of $\delta^{G S}$ is illustrated in table 5 . All the numbers of table 5 have been obtained by numerical minimization of (48) and by using the exact formula for $m_{3 / 2}$, but they fit very well the approximate expressions $(52,53)$. So we conclude that the results obtained for the simplest $\delta^{G S}=0$ case are essentially maintained for the general $\delta^{G S} \neq 0$ case.

So far we have considered only untwisted hidden matter. The most general situation, however, involves twisted matter. In fact, it can be argued on grounds of modular invariance that for $Z_{3}, Z_{7}$ orbifolds there cannot be two gauge groups with different $k^{-1} \beta$ coefficients and all the matter in the untwisted sector. The argument is very similar to that expounded in subsection 2.5. If only untwisted matter is present it is clear from (2) that $\frac{1}{16 \pi^{2}} \sum b_{i}^{\prime a}=\tilde{\beta}^{a}$, i.e. the complete beta function. Now, for $Z_{3}$ and $Z_{7}$ orbifolds the coefficient of $\log \left(\eta\left(T_{i}\right)\right)$ in eq.(1) must vanish, i.e. $b_{i}^{\prime a}=2 k^{a} \delta_{i}^{G S}$. Taking into account that $\delta_{i}^{G S}$ are universal for all the gauge group factors, this cancellation cannot take place unless all the gauge groups have the same $\left(k^{-1} \tilde{\beta}\right)$ factors, a situation which is useless 
for the practical purpose of fixing the dilaton since this is equivalent to having a unique condensate. For the rest of $Z_{N}$ orbifolds the restriction is not so strong, since all of them have an $N=2$ sector (the $Z_{6}$-II has two) associated with a fixed torus. In consequence there is a plane, say the $i=3$ plane, in which the previous cancellation does not take place. This means, in particular, that if for a $S U(N)$ factor there are singlet fields $A_{i}(i$ denotes a holomorphic index) giving mass to $M_{i}$ quarks (so $\sum_{i} M_{i}=M$ ), then the quantities $k^{-1}\left(N-M_{1}\right)$ and $k^{-1}\left(N-M_{2}\right)$ must be the same as in any other $S U(N)$ factor. In principle, there would be no restrictions on $M_{3}$.

Let us consider now gaugino condensation in the presence of twisted matter. Following similar steps to those of subsection 3.1 we work with an overall modulus $T$ and a generic $A$ field of the twisted type giving mass to $M(N+\bar{N})$ representations through a trilinear superpotential $W^{t r}=\sum_{\alpha=1}^{M} h(T) A Q_{\alpha} \bar{Q}_{\alpha}$ with $h(T) \neq$ const. In consequence, $\operatorname{det} \mathcal{M}=[h(T) A]^{M}$ in (28). Analogously, the perturbative piece of eq.(34) becomes now $T$-dependent, $W^{\text {pert }}=\hat{h}(T) A^{3}$. The $A$ field can be integrated out again through condition (32), leading now to

$$
A^{3}=\left(\frac{M}{3 \hat{h}(T)}\right)^{\frac{3 N}{3 N-M}}[h(T)]^{\frac{3 M}{3 N-M}}\left(32 \pi^{2} e\right)^{\frac{3(M-N)}{3 N-M}} \frac{e^{-24 \pi^{2} k S /(3 N-M)}}{[\eta(T)]^{6\left(\sum_{i} b_{i}^{\prime}-2 k \delta^{G S}\right) /(3 N-M)}}
$$

which substituted in $W=W^{n p}+W^{p e r t}$ gives a superpotential that depends on $S$ and $T$ only

$$
\begin{gathered}
W^{e f f}=\tilde{d}_{T} \frac{e^{-3 k S / 2 \tilde{\beta}}}{[\eta(T)]^{3\left(\sum_{i} b_{i}^{\prime}-2 k \delta^{G S}\right) / 8 \pi^{2} \tilde{\beta}}}, \\
\tilde{\beta}=\frac{3 N-M}{16 \pi^{2}}, \quad \tilde{d}_{T}=\left(\frac{M}{3}-N\right)\left(32 \pi^{2} e\right)^{\frac{3(M-N)}{3 N-M}}\left(\frac{M}{3 \hat{h}(T)}\right)^{\frac{M}{3 N-M}}(h(T))^{\frac{3 M}{3 N-M}} .
\end{gathered}
$$

This is very similar to eq.(47) for the untwisted case. In fact the similarity is even stronger since the Yukawa couplings $h(T), \hat{h}(T)$ must have modular weights $\left(-3-n_{A}-2 n_{Q}\right)$, $-3\left(1+n_{A}\right)$ respectively in order to preserve the correct modular weight of $W$. Therefore $h(T) \propto[\eta(T)]^{-2\left(3+n_{A}+2 n_{Q}\right)}, \hat{h}(T) \propto[\eta(T)]^{-6\left(1+n_{A}\right)}$ and, in consequence,

$$
W^{e f f} \sim \tilde{d} \frac{e^{-3 k S / 2 \tilde{\beta}}}{[\eta(T)]^{6-\left(3 k \delta^{G S} / 4 \pi^{2} \tilde{\beta}\right)}}
$$

where $\tilde{d}$ is defined in (37) and we have used eq.(2). Comparing with (47) we see that it is not likely that the presence of twisted matter substantially changes the results obtained for the untwisted case. It is remarkable how the Yukawa couplings generate the piece $\sim \eta(T)$ in the superpotential, even if this is not present at the beginning, as it occurs for 
$Z_{3}$ and $Z_{7}$ orbifolds. What is even more: the presence of twisted matter guarantees that the whole set of moduli appears in the final expression of $W^{e f f}$. This comes from a kind of completeness that exists between the moduli involved in the threshold corrections to $f_{W}$ (see eq.(11)) and those involved in the Yukawa couplings [27]. More precisely, the moduli appearing in the threshold corrections are those associated with the size and shape of the fixed tori of the orbifold, whereas the remaining ones always appear in the expression of the twisted Yukawa couplings. For instance, in the $Z_{3}$ and $Z_{7}$ constructions there are no threshold corrections at all, but all the twisted Yukawa couplings have a non-trivial dependence on the whole set of moduli.

\subsection{Other scenarios}

In all the phenomenologically interesting scenarios above described the perturbative interactions of the $A$ field (apart from its coupling to the quarks) play a crucial role. However, though less probable, it is also possible that those interactions are absent or that their contribution to the scalar potential is flat. Then the only non-trivial part of the superpotential is the non-perturbative one (28) and one may wonder whether there still exist interesting scenarios under these circumstances. For a unique condensate, as it was explained in subsection 3.1, this is not the case. The answer turns out to be afirmative, however, if the gauge group contains three or more subsectors. To see this, consider first a hidden sector $S U\left(N_{1}\right) \times S U\left(N_{2}\right)$ with matter content $M_{1}\left(N_{1}+\bar{N}_{1}\right)+M_{2}\left(N_{2}+\bar{N}_{2}\right)$ where the two condensates are "sharing" the same $A$ field in (28), i.e.

$$
W^{n p}=-N_{1}\left(32 \pi^{2} e\right)^{\frac{M_{1}}{N_{1}}-1} A^{\frac{M_{1}}{N_{1}}} \frac{e^{-\frac{8 \pi^{2}}{N_{1}} S}}{[\eta(T)]^{6-2 M_{1} / N_{1}}}-N_{2}\left(32 \pi^{2} e\right)^{\frac{M_{2}}{N_{2}}-1} A^{\frac{M_{2}}{N_{2}}} \frac{e^{-\frac{8 \pi^{2}}{N_{2}} S}}{[\eta(T)]^{6-2 M_{2} / N_{2}}}(58)
$$

Then the equation (32) (which, as it was argued in subsection 3.1, gives the minimum in A) has non-trivial solution

$$
A=\left(-\frac{M_{2}}{M_{1}}\right)^{\frac{N_{1} N_{2}}{N_{2} M_{1}-N_{1} M_{2}}}\left(32 \pi^{2} e\right)^{-1}[\eta(T)]^{-2} e^{-8 \pi^{2} \frac{N_{1}-N_{2}}{N_{2} M_{1}-N_{1} M_{2}} S}
$$

(notice that if the $A$ fields for the two subsectors are different each other the equation (32) has no solution, apart from the trivial one). Substituting (59) in $W^{n p}$ leads to

$$
W^{e f f}=D \frac{e^{-\alpha S}}{\eta^{6}(T)}
$$

\footnotetext{
${ }^{8}$ We choose here for simplicity $\delta_{i}^{G S}=0, k=1$, an overall modulus $T$ and untwisted matter fields.
} 


$$
\alpha=8 \pi^{2} \frac{M_{1}-M_{2}}{N_{2} M_{1}-N_{1} M_{2}}, \quad D=-\frac{N_{2}}{32 \pi^{2} e}\left(1-\frac{N_{1} M_{2}}{N_{2} M_{1}}\right)\left(\frac{-M_{2}}{M_{1}}\right)^{\frac{N_{1} M_{2}}{N_{2} M_{1}-N_{1} M_{2}}}
$$

The form of $W^{\text {eff }}$ is very similar to the pure YM case for a single condensate, see eq.(10). Consequently, the scalar potential does not have minima in $S$. However, if we had started with the two condensates of eq.(58) plus a third, pure YM, $S U\left(N_{3}\right)$ condensate, the corresponding effective superpotential would simply be

$$
W^{e f f}=D \frac{e^{-\alpha S}}{\eta^{6}(T)}+d_{3} \frac{e^{\frac{-8 \pi^{2}}{N_{3}} S}}{\eta^{6}(T)}
$$

where $D, \alpha$ are defined in (61) and $d_{3}$ is defined in (10). Then, as it was shown in subsection 2.3, the associated scalar potential develops minima at $2 S_{R} W_{S}^{\text {eff }}-W^{\text {eff }}=0$ and $T=1.23$ or any other value related to this by duality. It turns out to be many possibilities leading to reasonable values for the dilaton and the gravitino mass $m_{3 / 2}=$ $e^{K / 2}\left|W^{e f f}\right|$. Requiring $1 \leq S_{R} \leq 3.5,10^{2} \mathrm{GeV} \leq m_{3 / 2} \leq 10^{4} \mathrm{GeV}$ we find 72 scenarios of this type, which are given in table 6. Four of them satisfy $N_{a}>M_{a}$. Simplified expressions for $S_{R}, m_{3 / 2}$ can be obtained from the approximate condition $W_{S}^{e f f}=0$ (see eq. (22))

$$
\begin{aligned}
S_{R} & \simeq \frac{1}{\alpha-8 \pi^{2} / N_{3}} \log \left|\frac{D \alpha}{d_{3}\left(8 \pi^{2} / N_{3}\right)}\right| \\
m_{3 / 2} & \simeq \frac{1}{\left(2 S_{R}\right)^{1 / 2}\left(2 T_{R}\right)^{3 / 2}|\eta(T)|^{6}}\left|d_{3}\left(1-\frac{8 \pi^{2}}{N_{3} \alpha}\right)\right| e^{\frac{-8 \pi^{2}}{N_{3}} S}
\end{aligned}
$$

Using the previous expressions it can be seen that a realistic value for $m_{3 / 2}$ requires $S_{R} \simeq 0.34 N_{3} \simeq 0.32 \frac{N_{2} M_{1}-N_{1} M_{2}}{M_{1}-M_{2}}$.

Similarly, for a hidden sector with four condensing groups $G=\prod_{a=1}^{4} S U\left(N_{a}\right)$, $\sum_{a=1}^{4} M_{a}\left(N_{a}+\bar{N}_{a}\right)$ matter representations and two different $A$ fields associated with $S U\left(N_{1}\right) \times S U\left(N_{2}\right)$ and $S U\left(N_{3}\right) \times S U\left(N_{4}\right)$ respectively, the effective superpotential is

$$
W^{e f f}=D_{1} \frac{e^{-\alpha_{1} S}}{\eta^{6}(T)}+D_{2} \frac{e^{-\alpha_{2} S}}{\eta^{6}(T)}
$$

with obvious notation. In this case the number of different combinations giving $1 \leq S_{R} \leq$ $3.5,10^{2} \mathrm{GeV} \leq m_{3 / 2} \leq 10^{4} \mathrm{GeV}$ is much higher. This comes from the fact that there is a larger amount of parameters $\left(N_{1}, \ldots, N_{4} ; M_{1}, \ldots M_{4}\right)$ to play with. More precisely, there are 2702 cases from which 40 are displayed in table 7 . Approximate expressions for $S_{R}, m_{3 / 2}$ analogous to (63,64) can be straightforwardly written. In this case a reasonable value for $m_{3 / 2}$ requires $S_{R} \simeq 0.315 \frac{N_{2} M_{1}-N_{1} M_{2}}{M_{1}-M_{2}} \simeq 0.315 \frac{N_{4} M_{3}-N_{3} M_{4}}{M_{3}-M_{4}}$. 
The scenarios considered in this subsection are attractive in the sense that they are independent from the details of the perturbative superpotential for the singlet $A$ fields, provided this is flat, even though this does not seem to be the most usual situation.

\section{Conclusions}

We have studied in a systematic and modular invariant way a large class of string scenarios for which gaugino condensation in the hidden sector provides a potential source of supersymmetry breaking as well as a non-trivial scalar potential for the dilaton $S$, whose real part corresponds to the tree level gauge coupling constant $\left(\operatorname{Re} S=S_{R} \sim g_{\text {gut }}^{-2}\right)$. For the case of pure Yang-Mills condensation (i.e. no matter in the hidden sector) we find that no realistic results can emerge, even if the hidden sector gauge group $G$ is not simple: $G=\prod_{a} G_{a}$. Things change dramatically in the presence of hidden matter, which on the other hand, is the most frequent case. Then there arises a wide and very interesting class of scenarios with two hidden condensing groups $S U\left(N_{1}\right) \times S U\left(N_{2}\right)$ for which the dilaton dynamically acquires a reasonable value $\left(S_{R} \sim 2\right)$ and supersymmetry is broken at the correct scale $\left(m_{3 / 2} \sim 10^{3} \mathrm{GeV}\right)$. There is no need for fine-tuning to obtain good values for $S$ and $m_{3 / 2}$ simultaneously, actually it turns out that for constructions with Kac-Moody level 1 they are correlated. We have made an exhaustive classification of the working possibilities (see tables), giving also very simple approximate formulae for $S_{R}$ and $m_{3 / 2}$ which are useful to select models. Other types of working scenarios with three and four condensing groups have also been studied.

Remarkably, the results are basically independent from the value of $\delta^{G S}$ (the contributions from the Green-Schwarz mechanism). Furthermore, the minimization of the scalar potential also gives a value for the modulus $T\left(\operatorname{Re} T=R^{2}\right.$, with $R$ the radius of the compactified space) which in all cases is close to $T=1.23$. This indicates that the theory is spontaneously compactified at this very sensible scale, thus breaking target-space modular invariance in a spontaneous way. Concerning this point, it is worth noticing that Yukawa couplings induce a non-trivial dependence $\sim \eta(T)$ of the condensation superpotential on the whole set of moduli (see subsection 3.2.), even if this is absent at the beginning (as it occurs for $Z_{3}$ and $Z_{7}$ orbifolds, where there are no moduli-dependent threshold corrections).

\section{ACKNOWLEDGEMENTS}

We thank J. Louis for illuminating discussions. We also thank L. Ibañez and D. Lüst for very useful comments. The work of B.C. was supported by a Comunidad de 
Madrid grant.

\section{References}

[1] J. Ellis, S. Kelley and D.V. Nanopoulos, Phys. Lett. B260 (1991) 131; U. Amaldi, W. de Boer and H. Fürstenau, Phys. Lett. B260 (1991) 447; P. Langacker and M. Luo, Phys. Rev. D44 (1991) 817; R.G. Roberts and G.G. Ross, RAL-92-005 (1992)

[2] S. Dimopoulos, S. Raby and F. Wilczek, Phys. Rev. D24 (1981) 1681; L.E. Ibáñez and G.G. Ross, Phys. Lett. B105 (1981) 439; S. Dimopoulos and H. Georgi, Nucl. Phys. B193 (1981) 375; M. Einhorn and D.R.T. Jones, Nucl. Phys. B196 (1982) 475

[3] M. Dine and N. Seiberg, Phys. Rev. Lett. 57 (1986) 2625

[4] J.A. Casas, J. García-Bellido and M. Quirós, Nucl. Phys. B361 (1991) 713

[5] H.P. Nilles, Phys. Lett. 115B (1982) 193; S. Ferrara, L. Girardello and H.P. Nilles, Phys. Lett. 125B (1983) 457; J.P. Derendinger, L.E. Ibáñez and H.P. Nilles, Phys. Lett. 155B (1985) 65; M. Dine, R. Rohm, N. Seiberg and E. Witten, Phys. Lett. 156B (1985) 55; C. Kounnas and M. Porrati, Phys. Lett. B191 (1987) 91

[6] L. Dixon, talk presented at the A.P.S. D.P.F. Meeting at Houston (1990); V. Kaplunovsky, talk presented at the "Strings 90" workshop at College Station (1990); L. Dixon, V. Kaplunovsky, J. Louis and M. Peskin, unpublished

[7] J.A. Casas, Z. Lalak, C. Muñoz and G.G. Ross, Nucl. Phys. B347 (1990) 243

[8] A. Font, L. Ibáñez, D. Lüst and F. Quevedo, Phys. Lett. B245 (1990) 401; M. Cvetič, A. Font, L. Ibáñez, D. Lüst and F. Quevedo, Nucl. Phys. B361 (1991) 194

[9] S. Ferrara, N. Magnoli, T.R. Taylor and G. Veneziano, Phys. Lett. B245 (1990) 409

[10] H.P. Nilles and M. Olechowsky, Phys. Lett. B248 (1990) 268; P. Binétruy and M.K. Gaillard, Phys. Lett. B253 (1991) 119

[11] D. Lüst and T.R. Taylor, Phys. Lett. B253 (1991) 335

[12] B. de Carlos, J.A. Casas and C. Muñoz, Phys. Lett. B263 (1991) 248

[13] J. Louis, SLAC-PUB-5645 (1991)

[14] D. Lüst and C. Muñoz, CERN-TH.6358/91 (1991) 
[15] S. Ferrara, D. Lüst, A. Shapere and S. Theisen, Phys. Lett. B225 (1989) 363; S. Ferrara, D. Lüst and S. Theisen, Phys. Lett. B233 (1989) 147

[16] V. Kaplunovsky, Nucl. Phys. B307 (1988) 145; L. Dixon, V. Kaplunovsky and J. Louis, Nucl. Phys. B355 (1991) 649

[17] J.P. Derendinger, S. Ferrara, C. Kounnas and F. Zwirner, CERN-TH.6004/91 (1991)

[18] J. Louis, SLAC-PUB-5527 (1991); G. Lopes Cardoso and B. Ovrut, UPR-0464T (1991), UPR-0481T (1991)

[19] H.P. Nilles, Phys. Lett. B180 (1986) 240; M.A. Shifman and A.I. Vainshtein, Nucl. Phys. B359 (1991) 571; I. Antoniadis, K.S. Narain and T.R. Taylor, Phys. Lett. B267 (1991) 37; J.A. Casas and C. Muñoz, Phys. Lett. B271 (1991) 85

[20] I. Antoniadis, J. Ellis, A.B. Lahanas and D.V. Nanopoulos, Phys. Lett. B241 (1990) 24; S. Kalara, J.L. López and D.V. Nanopoulos, CTP-TAMU-69/91 (1991); S. Kelley, J.L. López and D.V. Nanopoulos, CTP-TAMU-105/91 (1991)

[21] N.V. Krasnikov, Phys. Lett. B193 (1987) 37

[22] T.R. Taylor, Phys. Lett. B252 (1990) 59

[23] E. Cremmer, S. Ferrara, L. Girardello and A. Van Proeyen, Nucl. Phys. B212 (1983) 413

[24] L. Dixon, J. Harvey, C. Vafa and E. Witten, Nucl. Phys. B261 (1985) 651, B274 (1986) 285

[25] J.A. Casas, E.K. Katehou and C. Muñoz, Nucl. Phys. B317 (1989) 171; J.A. Casas and C. Muñoz, Phys. Lett. B214 (1988) 63; A. Font, L. Ibáñez, H.P. Nilles and F. Quevedo, Phys. Lett. B210 (1988) 101

[26] S. Hamidi and C. Vafa, Nucl. Phys. B279 (1987) 465; L. Dixon, D. Friedan, E. Martinec and S. Shenker, Nucl. Phys. B282 (1987) 13; T.T. Burwick, R.K. Kaiser and H.F. Müller, Nucl. Phys. B355 (1991) 689; T. Kobayashi and N. Ohtsubo, DPKU9103

[27] J.A. Casas, F. Gómez and C. Muñoz, CERN-TH.6194/91 (1991)

[28] L. Dixon, V. Kaplunovsky and J. Louis, Nucl. Phys. B329 (1990) 27; L.E. Ibáñez and D. Lüst, CERN-TH.6380/92 (1992) 
[29] S. Mahapatra, Phys. Lett. B223 (1989) 47; Y. Katsuki, Y. Kawamura, T. Kobayashi, N. Ohtsubo, Y. Ono and K. Tanioka, Nucl. Phys. B341 (1990) 611

[30] L.E. Ibáñez, H.P. Nilles and F. Quevedo, Phys. Lett. B187 (1987) 25; Y. Katsuki, Y. Kawamura, T. Kobayashi, N. Ohtsubo, Y. Ono and K. Tanioka, Prog. Theor. Phys. 82 (1989) 171, DPKU-8904 (1989)

[31] S. Kalara, J. López and D.V. Nanopoulos, Phys. Lett. B269 (1991) 84

[32] I. Antoniadis, J. Ellis, J.S. Hagelin and D.V. Nanopoulos, Phys. Lett. B205 (1988) 459, B213 (1988) 56; A.H. Chamsadine and M. Quirós, Nucl. Phys. B316 (1989) 101

[33] J.A. Casas, A. de la Macorra, M. Mondragón and C. Muñoz, Phys. Lett. B247 (1990) 50

[34] J.A. Casas, M. Mondragón and C. Muñoz, Phys. Lett. B230 (1989) 63

[35] Y. Katsuki, Y. Kawamura, T. Kobayashi, Y. Ono and K. Tanioka, Phys. Lett. B218 (1989) 169

[36] T.R. Taylor, G. Veneziano and S. Yankielowicz, Nucl. Phys. B218 (1983) 493

[37] I. Affleck, M. Dine and N. Seiberg, Nucl. Phys. B241 (1984) 493

[38] D. Amati, K. Konishi, Y. Meurice, G.C. Rossi and G. Veneziano, Phys. Rep. 162 (1988) 169

[39] E. Witten, Phys. Lett. B155 (1985) 151; S. Ferrara, C. Kounnas and M. Porrati, Phys. Lett. B181 (1986) 263; M. Cvetič, J. Louis and B. Ovrut, Phys. Lett. B206 (1988) 227

[40] G.G. Ross, Phys. Lett. B211 (1988) 315

[41] V. Kaplunovsky and J. Louis, to appear

[42] L.E. Ibáñez, D. Lüst and G.G. Ross, Phys. Lett. B272 (1991) 251 


\section{FIGURE CAPTIONS}

Fig.1 Scalar potential $V$ versus $S_{R}=\operatorname{Re} S$ for a pure Yang-Mills $S U(8)_{k=1}$ hidden sector. $\operatorname{Im} S$ and $T$ have been set at their minimizing values. For other simple gauge groups the shape of the potential is similar.

Fig.2 Scalar potential $V$ (in logarithmic units chosen for convenience) versus $S_{R}=$ Re $S=g_{\text {string }}^{-2}, T_{R}=\operatorname{Re} T$ for a $S U(6)_{k=1} \times S U(7)_{k=1}$ hidden sector with $6(7+\overline{7})$ hidden matter representations and $\delta^{G S}=0$. $\operatorname{Im} S$ and $\operatorname{Im} T$ are set at their minimizing values. The two minima (related each other by duality) correspond to $S_{R}=2.1576$; $T_{R}=1.2345,0.8100$.

Fig.3 The same example as in fig.2, but for $\delta^{G S}=20 . Y / 2 \equiv S_{R}+\frac{\delta^{G S}}{8 \pi^{2}} \log \left(2 T_{R}\right)=g_{\text {string }}^{-2}$. The two minima correspond to $Y / 2=2.0588 ; T_{R}=1.2307,0.8125$. 